\title{
Focusing on Sexual Health Promotion to Enhance Preventive Behaviors among Gay Men and Other Men Who Have Sex with Men: Report from a State-of-the-Art Conference
}

\author{
Hilary Goldhammer • Kenneth H. Mayer
}

Published online: 25 February 2011

(C) Springer Science+Business Media, LLC 2011

HIV and other sexually transmitted diseases (STDs) continue to spread among gay men and other men who have sex with men (MSM) at concerning rates, despite public health efforts to decrease transmission $[1,2]$. This ongoing concentrated epidemic suggests that researchers and program specialists need to review which prevention interventions are working for MSM, and which are not. Using this information, we must develop more effective, culturally-tailored prevention interventions as well as ask new research questions. With these imperatives in mind, The Fenway Institute, Boston, MA (http://www.thefenway institute.org) convened the Sexual Health of Gay Men and Other Men Who Have Sex with Men (MSM): HIV/STD Prevention Plus Conference, in April 2010, bringing together 97 leading American researchers, community stakeholders, and public health officials to discuss the current state of knowledge on the sexual health of American MSM and the implications of these findings for HIV/ STD prevention programs and further research. We are grateful to have been given the opportunity to share many of the key reports and findings from the conference through this supplemental issue of AIDS and Behavior.

The conference was conducted at Fenway Health's conference center in Boston, MA, through the support of the National Institutes of Health's Office of AIDS Research, and the Centers for Disease Control and Prevention's

H. Goldhammer $(\varangle) \cdot$ K. H. Mayer

The Fenway Institute, Fenway Health,

1340 Boylston St, Boston, MA 02215, USA

e-mail: hgoldhammer@fenwayhealth.org

K. H. Mayer

Alpert School of Medicine/Miriam Hospital,

Brown University, Providence, RI, USA
National Center for HIV/AIDS, Viral Hepatitis, STD, and TB Prevention, with additional support from the Foundation for AIDS Research (amfAR) and John Snow, Inc. Participants hailed from academic institutions, community organizations, and public health departments across the United States.

Although the meeting covered a wide range of topics related to MSM sexual health, a few dominant themes emerged. First, we heard that most of the work in HIV prevention has focused on individual behavior without taking into account larger social forces, such as policies, institutions, cultural norms, and other contexts that shape and interact with identity and behavior. An increasing number of studies, however, are starting to look at these contexts with the hope that they can be harnessed for improved prevention interventions. Second, we discussed the tendency in HIV prevention, as in all of public health, to aim our goals toward diminishing negative behaviors rather than promoting health positive behaviors. Several presenters argued for designing interventions that build upon the assets of MSM individuals and communities, rather than only focusing on deficits. Third, we challenged each other to find ways to reduce the cultural stigma associated with gay identity and behavior, and to directly address its impact on gay men. Finally, we established that gay, bisexual and other MSM's health needs cannot and should not be reduced to a single disease (i.e., not just HIV); rather, we must step back and consider all aspects of MSM sexual health, including improved mental health and development of a positive sexual identity, access to civil rights, and the ability to achieve intimacy, pleasure, and nurturing relationships.

The intent of this journal supplement is to share several of the research reports and commentaries from the meeting in the hope that they will inform future practice and policy, 
and that they will stimulate researchers and public health officials to develop new initiatives to close gaps that were identified during the meeting. In order to give the reader a sense of the entire scope and flow of the meeting, the meeting agenda and summary of presentations are given below.

\section{Summary of Presentations}

\section{Day One: Opening Remarks}

The first day of the meeting opened with welcoming remarks given by Kenneth Mayer, MD, co-chair of The Fenway Institute; Jonathan Mermin, MD, MPH, Director of the Division of HIV/AIDS Prevention, CDC; and Kevin R. Frost, CEO of amfAR. Each speaker's comments were designed to challenge the group to carefully and critically review what is known about MSM sexual health, what is missing, and what needs to be done.

\section{What Is Sexual Health?}

Eli Coleman, PhD (University of Minnesota Medical School) opened the session by discussing historical definitions of sexual health by major international health organizations and how these definitions have evolved. The basic ingredients for sexual health are now considered to be: healthy physical and mental development; development of a positive sexual identity; development of the capacity for intimacy; an environment that promotes sexual health; and recognition of sexual health as a basic human right. Dr. Coleman emphasized that now is a critical moment in time for the promotion of MSM sexual health because both the public health and health care communities have begun to recognize that sexual health is not just about safer sex and the absence of disease, but is much more holistic, and includes relationships, spirituality and overall well-being. Coleman and colleagues have developed a Sexual Health Model for HIV prevention and have applied it to MSM.

Jim Pickett (AIDS Foundation of Chicago) argued that if researchers, public health officials, and community leaders want to make a difference in the sexual health of gay men, we must shift our perspective from one of crisis, disease, and personal failures (a formula that is not working well), to one that is holistic, multicultural, asset-driven, relationally-focused, empowering and celebratory. These are some of the foundational principles of the new Gay Men's Health Agenda, which seeks to improve the health and lives of gay men by tapping into community activism forces and by making recommendations to policymakers and researchers.
Social Determinants and Ecosocial Theory:

The Socio-Cultural Contexts of Stigma, Marginalization, and Structural Inequalities

Leo Wilton, PhD (SUNY Binghamton) focused on the need to better understand the socio-cultural contexts of sexual health that have an impact on the disparate HIV/STD infection rates among gay men of color. The concepts of stigma, marginalization, and structural inequalities can provide a theoretical framework to examine the complexities of the AIDS epidemic, as situated in the everyday, lived experiences of gay men of color.

Sexual Pleasure and Intimacy: How Do We Include Them in HIV Prevention?

Alex Carballo-Diéguez, PhD (Columbia University; NY State Psychiatric Institute) presented preliminary data from his qualitative research on why some MSM engage in intentional condom-less sex (aka "barebacking") despite its known risks. Study participants reported that barebacking greatly increased pleasure and satisfaction, provided more emotional intimacy, and could sometimes act as an expression of love.

Social, Cultural, and Structural Factors that Influence Sexual Health and Behavior in Different Racial/Ethnic Groups

Frank Wong, PhD (Emory University) presented preliminary findings from his current research on how sociocultural characteristics influence sexual health and mental health in a national sample of self-identified gay men/MSM of Asian and/or Pacific Island ethnicity. Preliminary regression analyses found that Asian men who identify more strongly as American were also more likely to be "out" to their friends, co-workers, and families. They were also more likely to feel as if they were part of the gay communities in their cities. Nativity may turn out to be more important than ethnicity as an influencing factor on sexual health outcomes among MSM of Asian descent.

Gary Harper, PhD, MPH (DePaul University) shared findings from his studies on the socio-cultural influences that have an impact on the sexual health of young Black gay and bisexual men and other Black MSM. He described how different communities (e.g., gay, black, mainstream, family) can influence the human life values, connectedness, intimacy, and future life options of young Black men. Many of these influences are negative; for example, young Black MSM are often sexually objectified by the gay community, lacking in role models, and rejected by friends and family and the Black community because of their sexual orientation. Harper recommends that interventions 
be culturally grounded and based in the lived experiences of diverse groups of gay and bisexual men and other Black MSM.

Isa Fernandez, PhD (Nova Southeastern University) presented key findings from her mixed methods study of social and cultural influences on sexual and drug use behaviors of Latino men in South Florida. Among the quantitative findings, psychosocial distress and higher levels of "Americanism" were found to be associated with unprotected anal sex among Latino men who were foreign born. From her qualitative research sample, it was found that negative cultural and societal messages about homosexuality can lead to isolation, secrecy, and shame, while strong cultural influences and conflicting messages from Hispanic, mainstream American and gay culture contribute to risk and protection. The experience of poverty and immigration can exacerbate these conditions.

Social Dynamics, Sexual Networks, Neighborhood Characteristics, and Urban Migration

Carl Latkin, PhD (Johns Hopkins Bloomberg School of Public Health) presented baseline findings from his intervention research on using social networks to improve the sexual health and well-being of Black MSM in Baltimore, among whom HIV rates are alarmingly high. In his baseline findings, Latkin discovered that many Black MSM have female partners, and that they talk to network members about their same sex behavior, but do not talk about condoms. Latkin and his study partners are still exploring questions about how social networks can be identified, how they can be tapped for changes in social norms, and whether this is even effective for the people in these networks.

Beryl Koblin, PhD (New York Blood Center; Mailman School of Public Health, Columbia University) discussed the ways in which neighborhood factors can influence individual health and presented some preliminary qualitative findings from a new study to identify key neighborhood-level characteristics that may influence sexual risk behaviors, substance use, and depression among 1,500 gay men in New York city. If it is found that neighborhood factors matter for at-risk MSM, then interventions could attempt to address factors on multiple levels; for example, interventions could decrease levels of neighborhood homophobia while strengthening social ties and social identity at the group and individual levels.

Steven Kurtz, PhD (Center for Drug and Alcohol Studies, University of Delaware, Coral Gables, FL) shared findings from his research on migration and sexual and drug risk behavior among MSM living in South Florida cities. Kurtz and colleagues have developed a theory that men migrate to gay-friendly areas such as South Florida in order to find friendship, acceptance, and freedom from shame and family judgment, but often end up losing the social capital from their hometowns and becoming immersed in a culture that celebrates drug use and hypersexuality. Potential outcomes related to this migration experience are social alienation, substance abuse, and HIV/ STDs. Quantitative data from Kurtz's sample of high-risk men seems to support this theory and suggests that migration to South Florida may increase sexual risk behavior while decreasing positive coping behaviors. Kurtz and colleagues have developed a group session intervention that helps MSM strengthen their resilience, from more intimate and lasting relationships, and achieve life goals.

Risk and Resilience in MSM; Addressing Mental Health within the Context of Sexual Health Promotion

Ron Stall, PhD, MPH (Graduate School of Public Health, University of Pittsburgh) challenged the current "deficits" focus taken by most HIV interventions and proposed using a more strengths-based approach to intervention design for gay and bisexual men. Stall suggested that researchers study the men who have overcome drug use and who have remained disease free despite having a high-risk profile. He also recommended looking at community mobilizing patterns that strengthen community interactions, and studying how civil rights laws (e.g., marriage and employment laws) affect mood and behavior in lesbian, gay, bisexual, and transgender (LGBT) people.

Steve Safren, PhD (The Fenway Institute; Massachusetts General Hospital) summarized syndemic research on MSM, explaining that the greater the number of comorbid mental health problems, the greater the odds of sexual risk behaviors. Logically, the promotion of sexual health in MSM should account for complex mental health comorbidities; moreover, integrating the treatment of mental health problems within HIV prevention interventions has the potential to markedly boost the effectiveness of those interventions.

\section{MSM Partnerships}

Colleen Hoff, PhD (San Francisco State University) noted that about $50 \%$ of gay men are in committed relationships, and on average, half of those in relationships allow sex with partners outside the relationship. Hoff performs research on the agreements couples make about monogamy versus non-monogamy and the rules they set for sex with outside partners. She has found that agreements are very common and can be protective. Feelings of equality in the relationship, as well as getting HIV-specific support from the primary partner lowers the risk of engaging in unprotected anal intercourse with non-primary partners. Hoff recommends that couples' interventions account for the 
influence partners have on each other and use it as a synergistic tool.

Gilbert Herdt, PhD (San Francisco State University) provided data that illuminated the physical health, mental health, and social support benefits of being in a satisfying legal marriage for heterosexuals and LGB persons. He also delineated the negative effects of "marriage denial" by states, such as reinforcing stigma, exacerbating family and community rejection, and subtly supporting sexual risk taking. Most LGB people aspire to marriage, according to a recent national poll, but the majority of states do not support it. At the same time, gay men in marriages face greater stressors to their relationships due to lack of community recognition, lower socioeconomic status, lower family support, and destabilization of the legal status of their marriage (including lack of federal recognition).

\section{Young Men Who Have Sex with Men}

Frank Floyd, PhD (Georgia State University) presented research on the "coming out" experience among MSM youth, including data on the sequence of milestones experienced when coming out (awareness of same-sex attraction, same-sex sexual experimentation, self identification as gay or bisexual, and identity commitment/disclosure). He reported that parental attitudes can influence sexual orientation identity development and healthy behaviors, and that coming out can affect relationships with parents, ranging from no effect (24-31\%) to creating distance $(13 \%)$ to ruining the relationship $(3 \%)$.

Gary Harper, PhD, MPH (DePaul University) discussed the developmental, cultural, and contextual considerations for HIV prevention among gay/bisexual male youth. From these considerations, he concluded that interventions need to acknowledge that sexual risk and protective behaviors are multi-faceted and impacted by a range of social, cultural, economic, and political forces; that interventions need to explore the role of historical, psychological, and structural factors that influence sexual risk and protective behaviors; and that interventions need to acknowledge and attend to the unique cultural and contextual factors that influence the sexual lives of gay and bisexual adolescents of color.

The Role of New Information and Effective HIV Medications on Sexual Health: Seroadaptive Behaviors, Therapeutic Optimism, and Viral Load Beliefs

David Purcell, JD, PhD (Division of HIV/AIDS Prevention, CDC) discussed changes in attitudes, cognitions, and beliefs among MSM since the advent of HAART, specifically with regard to sexual protective and risk behaviors. There is evidence that "treatment optimism" (the belief that getting HIV is not that bad because there are drugs to manage it) is associated with higher sexual risk in the general MSM population, although not among HIV-infected MSM. There is also evidence that seroadaptive behaviors, such as serosorting (having unprotected sex only with partners who are believed to have the same HIV serostatus), can reduce risk compared to having indiscriminate unprotected sex. Some of the implications for MSM's sexual health include the need to address seroadaptive behaviors and treatment-related attitudes and cognitions in new interventions, and the need to educate medical providers about these issues to ensure they can effectively communicate the risk levels to their patients.

\section{The Role of New Media on Sexual Health}

Simon Rosser, PhD, MPH, LP (School of Public Health, University of Minnesota) provided an overview of gay/ bisexual men's use of the Internet for sex, and gave recommendations for developing better online HIV prevention interventions based on current research. Attrition has been a large problem for online risk-reduction interventions; therefore, Rosser recommends (based on his research) using image-laden, sexually explicit, game-oriented interventions that also offer small compensation. On-line activities that include critical self-reflection appear to decrease risk behaviors, at least in the short-term.

David Novak, MSW, LICSW, CAS (Online Buddies, Inc.; OLB Research Institute) shared the sexual health promotion and research activities implemented by Online Buddies, Inc. (OLB) Research Institute over the last several years. OLB owns and operates social and sexual networking sites for MSM. In 2004 they developed a health promotion and research department that uses Internet innovation to address critical sexual health issues. Their guiding principles are: to help gay men and other MSM have the best sex lives with the least harm; to educate gay men and other MSM about sex and the law, with a focus on HIV/STD stigma and discrimination; and to use scientific evidence to further understand and implement structural interventions across sites and inform industry peers of their findings.

Epidemiological Trends in HIV and STD Prevalence and Incidence in American MSM; Behavioral and Sexual Risk-Taking Trends among MSM

Richard Wolitski, PhD (Division of HIV/AIDS Prevention, CDC) reported that MSM are 44 times as likely to have an HIV diagnosis as other men, and that young Black MSM bear a disproportionate burden of HIV infection. The incidence of syphilis and gonorrhea appear to be rising among MSM, and utilization of prevention services for 
MSM, such as HIV testing and vaccination against Hepatitis A and B is not optimal. Unprotected sex among MSM is increasing, but the reasons behind the increase are not fully understood.

\section{Substance Use and Sexual Risk-Taking Trends}

Grant Colfax, MD (San Francisco Department of Public Health) reported that there continues to be a high prevalence of substance use among MSM and that non-injection drug use is associated with increased HIV risk. Some reasons for this association are theorized to be: altered mental state leads to reductions in condom use; and enhanced desire/pleasure and decreased pain lead to more partners, longer sex, and tissue damage/blood contact. Interventions should: (1) address (poly)substance use and risky sexual behavior together; (2) target MSM groups specifically; (3) take into account episodic use versus dependence; and (4) explore pharmacotherapies.

\section{Day Two: Opening Remarks}

The second day of the meeting opened with welcoming remarks from Stephen Boswell, MD, CEO of Fenway Health.

Effectiveness, Dissemination, and Implementation of HIV Prevention Interventions

Greg Rebchook, PhD (University of California, San Francisco) provided an overview of the successful implementation, adaptation and dissemination of the Mpowerment Project (MP), a community-based intervention for young MSM that has been adapted by over 70 communities. MP takes a multi-level, community-level approach that addresses personal, inter-personal, social and communitylevel issues facing young gay/bisexual men. Each program's issues and activities are determined by the participants themselves and the ideas are turned into events and/ or outreach materials to reach their peers. Successful adaptation is associated with funding level, staff buy-in of guiding principles, and understanding and integration of the socio-cultural needs of the diverse communities served.

Kevin Cranston, MDiv (Massachusetts Department of Public Health) reported that although HIV incidence has decreased in Massachusetts, the percentage of cases due to MSM sexual contact has grown. Massachusetts has had success with free condom distribution and implementation of evidence-based interventions (EBIs), particularly among non-White and non-Boston MSM, but it is not clear if it is better to have widespread dissemination or to only target those most at risk. The state is working towards a more broadly focused sexual health agenda that does not just focus on risk, that considers intimacy in friendships and romantic/sexual relationships among MSM, and that is flexible enough to evolve along with the MSM communities.

Matthew Golden, MD, MPH (University of Washington; Public Health: Seattle and King County) examined the current landscape for local HIV prevention interventions and their funding, specifically in Seattle/King County, WA. Current levels of funding are not adequate for supporting scalability of evidence-based interventions or other interventions, particularly since there is a trend toward increasing support for case-finding and treatment. A shift to a sexual health focus has the benefit of being higher yield, but is risky as a long-term strategy since there is not yet evidence that it will work.

Darrell Wheeler, PhD, MPH, MSW (Hunter College School of Social Work) discussed the effectiveness, dissemination, and implementation of prevention interventions for Black men, noting that Black MSM are at disproportionate risk of $\mathrm{HIV}$, and that historical racism, stigma, and medical negligence/harm towards people of color play a unique role. There are many cultural and structural factors worth exploring, including poverty, racism, housing, incarceration, and homophobia; interventions should target these areas in addition to behavioral factors. In addition, there is a need for community anchored services that build on relationships established with men over time to ensure that basic needs (housing, income) as well special needs of men are appropriately assessed, and are linked to mental health, substance use, social service, and medical services.

Ilan Meyer, PhD (Mailman School of Public Health, Columbia University) reported startling statistics regarding the disproportionate percentage of Black MSM who become HIV-infected and commented on how this is a true crisis that must be addressed. He discussed how minority stress, including internalized homophobia, can be compounded by racism in MSM communities, and is associated with increases in risk-taking, lower adherence to HIV medications, and lower awareness and participation in prevention interventions. At the same time, resilience and coping factors that seem to protect black LGBT people from the effect of racism and homophobia may be mobilized for HIV prevention.

Engaging Community-Based Organizations and Clinical Providers to Promote Sexual Health

Steve Gibson, MSW (Magnet; San Francisco AIDS Foundation) talked about the successes and challenges of his community-based organization, Magnet, which is a gay men's health and community center offering integrated sexual health services and community events in a storefront 
located in San Francisco's Castro neighborhood. Although Magnet serves a large number of men in San Francisco, gaps in sexual health services and education still exist in the city, including the need for: greater access to HIV testing, an increase in understanding and dissemination of information on serosorting and strategic positioning, education on syphilis screening for primary care providers, and a better understanding of the sexual health needs of HIVinfected gay men.

Marjorie Hill, PhD (Gay Men's Health Crisis) discussed the need to address social determinants of health, such as poverty, racism, and stigma in approaching HIV prevention through community organizations and advocacy. The Gay Men's Health Crisis (GMHC) takes a broad approach to advocacy through tactics such as giving testimony and briefings to government officials, and publishing policy papers that provide up-to-date research, program challenges, and policy change ideas (sample topics include HIV and aging, the FDA blood ban, and defunding abstinence only programs). GMHC also uses social marketing techniques that focus on positive relationships between men and between gay/bisexual youth and their families.

Alex Gonzalez, MD, MPH (Fenway Health) spoke about how medical providers can have a positive effect on sexual behavior through talking effectively with their MSM patients. Providers can create welcoming environments for LGBT patients by posting non-discrimination policies and by training staff in LGBT cultural competency. During the patient interview, providers should ask about sexual behavior in a routine manner without making assumptions about identity or using labels, and should assure confidentiality and trust. They should be prepared to offer condoms and to demonstrate proper use on a model, and should help patients set up realistic self-management goals.

New Prevention Technologies: What Is Likely to Be Available in the Near Term, What Needs to Be Considered to Enhance Implementation and Uptake, and Debate Around Preparing for Roll-Out

Bernard Branson, MD (Division of HIV/AIDS Prevention, National Center for HIV/AIDS, Viral Hepatitis, STD, and TB Prevention, CDC) briefly reviewed the benefits and drawbacks of some newer methods of HIV testing, including oral fluid and home self-testing. He then presented data showing the need for a test that can detect acute infection within 10 days of exposure, and provided promising data on new laboratory tests for detecting acute infection, including the development of point-of-care tests for earlier detection of new infections.

Ian McGowan, MD, PhD, FRCP (University of Pittsburgh School of Medicine; Magee-Womens Research Institute) provided the rationale for developing rectal microbicides (condom use is not consistent; lubricants are often used anyway) and presented data on rectal safety and acceptability studies. The next steps are to continue developing rectal specific products and applicators, and to begin Phase 2/2B studies.

Susan Buchbinder, MD (San Francisco Department of Public Health; University of California, San Francisco) discussed the current trials being done on pre-exposure prophylaxis (PrEP) that will help determine its short-term biomedical safety, behavioral safety, and pill-taking patterns. Only one trial, however, is studying MSM, and therefore generalization to all MSM populations will not be understood yet. Buchbinder also described the program Prevention Umbrella for MSM in the Americas (PUMA), which aims to select interventions likely to have the greatest impact on a population and then obtain community input on components of a prevention package so they can build an efficacy trial of a menu-based HIV prevention package.

Steve Wakefield (HIV Vaccine Trials Network; The Legacy Project) discussed the challenges of communicating complex scientific research to communities at risk of HIV infection. Over the years, researchers have learned to incorporate the values and concerns of communities into study design and operation of trials, but still have a great deal of work to do to improve capacity to ensure dissemination of results to the community, and to value the impact on a range of stakeholders with respect to culture or ethnic diversity.

How the USPHS Can Enhance MSM Sexual Health Programmatically, and What Kinds of New Research Initiatives Are Warranted

Dianne Rausch, PhD (Center for Mental Health Research on AIDS, NIMH) presented several of the HIV prevention research priorities for NIMH, including but not limited to focusing on safer sexual behavior within the context of sexual pleasure in order to increase effectiveness and longterm sustainability, integrating behavioral change interventions into disease management for HIV-infected persons, focusing on developmental, cultural and societal influences, intersecting biomedical with behavioral interventions, and targeting structural change. Rausch also discussed NIMH's strategies to partner with various federal and community partners, including CDC's prevention with positives programs, the HIV trials networks, and state health departments.

Carl Dieffenbach, PhD (Division of AIDS, NIAID) gave a broad overview of the research priorities for the $\mathrm{NIH}$, explaining how $\mathrm{NIH}$ and $\mathrm{CDC}$ are involved in a national HIV/AIDS prevention strategy with the overarching goals to reduce incidence, improve access to care, 
improve health outcomes, and reduce health care disparities. Dr. Dieffenbach explained that the field is currently at a crossroads in HIV prevention with the advent of new technologies and other interventions that are being proven by evidence, but now need to be sustainable, scalable, and able to be done in partnership with local agencies, both public and private. Moreover, he urged the use of assetsbased models that respect the full and active lives of individuals, that provide tools for people to use, and that integrate housing and other life needs with health care delivery.

Kevin Fenton, MD, PhD (National Center for HIV/ AIDS, Viral Hepatitis, STD, and TB Prevention, CDC) shared the CDC's shift in perspective and strategic plan from an HIV disease focus to a more holistic, sexual health framework. Using a new sexual health frame provides an opportunity to have frank, honest discussions not only about health outcomes but the behaviors and social contexts that are driving the disease. The CDC has developed a green paper on sexual health with key objectives and a range of strategies to implement those objectives in MSM and other communities.

Laura Cheever, MD, ScM (HIV/AIDS Bureau, HRSA) talked about HRSA's recent and current projects that relate to gay men's health, including the successful Stop Bullying Now campaign for adolescents that has a focus on LGBT youth, and an initiative focused on HIV-infected young men of color that was only partially successful due to some programs' inability to reach the target audience. In terms of the future, Cheever noted that HRSA's funding has increased for community health centers, offering an opportunity for more growth in LGBT and HIV interventions there. In addition, she asked the audience to help push for more funding for Ryan White and for programs that reach LGBT youth, particularly those of color.

\section{What States Can Do to Enhance MSM Sexual Health Programmatically}

John Auerbach, MBA (Massachusetts Department of Public Health), who is the current leader of the Association of State and Territorial Health Officials, remarked that health programs for LGBT populations are uneven from state to state and generally limited to infectious disease. Auerbach, however, sees potential for growth, since health departments tend to be more progressive, rely more on federal funding, and can embed programs within their departments. Massachusetts has been a leader in this area by collecting health data on LGBT populations, improving violence and injury programs that address LGBT people, forming an advisory board to look at how substance abuse services address LGBT issues, and changing all vital records to include LGBT relationships.
Murray Penner, BS (National Alliance of State and Territorial AIDS Directors) shared his thoughts on what state health departments can do to address gay men's health using a more holistic, less disease-specific framework. Although the political landscape is supportive right now, the fiscal crisis has meant severe budget cuts in HIV funding. Other challenges include reaching at-risk populations and funding interventions that are politically risky. He recommends that communities tap into the sympathetic political leadership, that researchers and health departments collaborate more, and that everyone expands their "tool box" for interventions to ensure it offers a large variety of options to meet individuals' needs.

What Are the Next Steps Forward to Promote MSM Sexual Health and to Continue the Discussion in the Wider Community?

Stewart Landers, JD, MCP (John Snow, Inc) presented the priorities of the Gay Men's Health Agenda and a framework for moving forward. Some of the priorities include funding and expanding social behavioral and biomedical research; developing and funding data collection on sexual orientation and gender identity federally; funding campaigns to combat homophobia, biphobia and transphobia; eliminating "no promo homo" [Section 2500 of the federal Public Health Service Act 300ee (b), (c) and (d)]; and creating an office of LGBT Health at the US Department of Health and Humans Services. Strategies for moving forward include: supporting local social marketing and better tracking of local activities using global MSM web blasts as a model; adopting a framework of overall health and wellness utilizing an assets-based approach; supporting men's health summits; and implementing translational research designed to take what we know about HIV prevention and expand it to targeted communities. The community can organize to nurture leadership, take ownership of its own health, weave health into community activities, acknowledge diversity within the community, and take a broader view of sexual health.

Leo Rennie (American Psychological Association; National Black Gay Men's Advocacy Coalition) discussed the ways in which the American Psychological Association (APA) is promoting the sexual health of MSM (e.g., by advancing psychology as a means of improving the health and well-being of LGBT people, as a means of increasing understanding of gender identity and sexual orientation as aspects of human diversity, and as a means of reducing stigma, prejudice, discrimination, and violence toward LGBT people). He also presented the National Black Gay Men's Advocacy Coalition (NBGMAC) federal policy priorities, including improvement and expansion in care service, research, and programs to address Black gay men's 
health needs, such as HIV prevention and substance abuse; monitoring the establishment of the National Institute for Minority Health and Health Disparities at NIH; and monitoring health care reform implementation's impact on gay men's sexual health.

\section{What Have We Learned? Where Do We Go from Here?}

Stephen Morin, PhD (University of California, San Francisco), who served as a rapporteur for the meeting, highlighted many of the predominant themes of the meeting, including the need to reduce stigma and its impact on gay men, especially young gay men, and to help these youth develop a positive identity; the need to focus on the whole person and not just disease; and the need to develop new community HIV prevention norms that go beyond condom use since these norms are weakening (e.g., serosorting, new technologies). Morin remarked on the optimism during the meeting, and how this needs to be spread to mobilize communities. He also pointed out the challenges of scaling up, but was encouraged by the new national strategies being developed.

Judy Auerbach, PhD (San Francisco AIDS Foundation), who also served as a rapporteur, began with a one-sentence take-away from the meeting: "how to better understand and address the whole man in his whole environment over his whole life in an assets-based frame without devolving into reductionism." What is needed, she continued, are multilevel, multi-component, multi-discipline strategies. Although the complexity of this challenges us, we cannot revert back to what we are comfortable doing, and, as this meeting has suggested, we need to better find individual and contextual factors and hypothesize more clearly about how they operate to confer resiliency and vulnerability. A number of constructs came up during the meeting, including gender and sexuality; race and ethnicity; risk, vulnerability, and resilience; mobility (migration, immigration, forced dislocation); historical oppression and marginalization and liberation; and settings and institutions and venues. From these we should build conceptual models that incorporate more theory, and we should engage more with other disciplines, such as the humanities, business and marketing. Finally, we should not just engage with the community after the fact, but should start with hearing from communities in some systemized fashion about what is perceived to be needed and meaningful and then derive our questions for analysis.

\section{Conclusions}

The meeting brought together a diverse group of researchers, public health officials, clinicians and community activists who presented new and provocative data and commentary. As the HIV/AIDS epidemic among MSM heads into its fourth decade in the United States, with scant signs of abating, all agreed that new approaches are needed. The articles in this monograph distill the ideas presented at the meeting, and suggest several ways forward. Overall, there was consensus that learning how to best promote healthy practices and studying those MSM who have been resilient in the face of multiple adversities, and who have steadfastly maintained healthy behaviors or successfully recovered after being risky, should offer new insights that can help the development of more culturallyappropriate and effective programs. Many questions remain as to how to best create assets-based programs, and how to link emerging biomedical interventions, like antiretroviral chemoprophylaxis, with evidence-based behavioral approaches. The challenges are great, but worthy of our best efforts. It is our hope that by sharing this series of papers that emerged from the meeting, we will continue the discussion, thus facilitating the development of new holistic approaches that may finally arrest the unmitigated spread of HIV among MSM.

Acknowledgments The editors wish to acknowledge the efforts of the Organizing Committee for this meeting: Judith D. Auerbach, Cornelius Baker, Kendall J. Bryant, David Burns, Alex CarballoDiéguez, Grant Colfax, Carl W. Dieffenbach, Brian Feit, Andrew D. Forsyth, Robert Garofalo, Matthew Golden, Cynthia Grossman, Matthew Hogben, Richard A. Jenkins, Kent Klindera, Stephen F. Morin, Susan F. Newcomer, Jim Pickett, David Purcell, Steven Safren, Steve Shoptaw, Ron Stall, Carlos Toledo, Sten H. Vermund, Steve Wakefield, Darrell P. Wheeler, Richard Wolitski, and Sheryl Zwerski. The editors also want to acknowledge the assistance of key members of The Fenway Institute: Rodney VanDerwarker, Susan Johnson, Tom Kane, Mark Hooker, and Mark Fallon. We are grateful for the support provided by the National Institute of Health's Office of AIDS Research, the Centers for Disease Control and Prevention, the Foundation for AIDS Research (amfAR) and John Snow, Inc. Finally, we would like to thank Dr. Seth Kalichman and the staff of AIDS and Behavior for their assistance in the editing of this monograph.

\section{References}

1. Hall HI, Song R, Rhodes P, et al. Estimation of HIV incidence in the United States. JAMA. 2008;300(5):520-9.

2. El-Sadr WM, Mayer KH, Hodder SL. AIDS in America-forgotten but not gone. N Engl J Med. 2010;362:967-70. 